\title{
Article \\ Effect of Global and Local Refutation Mechanism on Rumor Propagation in Heterogeneous Network
}

\author{
Liang'an Huo * and Yuqing Zhang
}

check for updates

Citation: Huo, L.; Zhang, Y. Effect of Global and Local Refutation Mechanism on Rumor Propagation in Heterogeneous Network. Mathematics 2022, 10, 586. https:// doi.org/10.3390/math10040586

Academic Editors: Davide Valenti and Roman Starosta

Received: 17 December 2021

Accepted: 27 January 2022

Published: 14 February 2022

Publisher's Note: MDPI stays neutral with regard to jurisdictional claims in published maps and institutional affiliations.

Copyright: (C) 2022 by the authors. Licensee MDPI, Basel, Switzerland. This article is an open access article distributed under the terms and conditions of the Creative Commons Attribution (CC BY) license (https:/ / creativecommons.org/licenses/by/ $4.0 /)$.
Business School, University of Shanghai for Science and Technology, Shanghai 200093, China; 192891049@st.usst.edu.cn

* Correspondence: liang_anhuo@usst.edu.cn

\begin{abstract}
In the process of rumors propagation, the behavior of individuals to propagation or refutation is not only influenced by the surrounding global environment but also the local environment. In this paper, a modified rumor propagation model is proposed considering the global and local effects of the rumor refutation mechanism and the activity rate of individuals in a heterogeneous network, and the dynamics process of the rumor propagation is derived by using the mean-field equation. Combining theoretical proving and simulation analysis, it shows that the critical threshold of rumor propagation has a great relationship with individual activity rates and global and local effects of the rumor refutation mechanism. The greater the government's efforts to refute rumors, the lower the critical threshold of rumor propagation and the smaller the final rumor size. While relatively, people are much more likely to believe the global rumor refutation information taken with official information, local rumor refutation information has little influence on rumor propagation.
\end{abstract}

Keywords: rumor propagation; refutation mechanism; critical threshold; activity rate; heterogeneous network

\section{Introduction}

Rumors are unconfirmed statements that may cause adverse effects on our society's stability [1,2]. With the rapid development of social networks, the spread of rumors has become faster, and its impact is more widespread. In order to reduce the negative impact of rumor propagation, the government has invested a lot of resources in refuting rumors to control the spread of rumors. For example, the recent spread of the COVID-19 has caused a lot of rumors about the epidemic, and some of them have seriously affected people's life and social stability $[3,4]$. Fortunately, the government implements the rumor refutation strategies and promptly announces authoritative information to the public, which can greatly reduce the negative impact of rumors on society globally. Moreover, the spontaneous rumor refutation strategies of non-governmental organizations (NGOs) have a certain inhibitory effect on the spread of rumors locally. With the joint efforts of the government and some NGOs, the spread of rumors will be effectively controlled. Therefore, it is necessary for us to study the global and local influence of the rumor refutation mechanism to better guide public opinion.

As early as the 1960s, researchers began to study the mechanism of rumor propagation. Daley and Kendal comparatively studied the similarity between the spread of epidemics and rumors and proposed the DK model, which revealed the differences between the two spread mechanisms [5]. The MK model proposed by Maki and Thompson suggested that rumors were spread through direct contact between spreaders and other individuals [6]. With further research on complex network models, Newman et al. believed that the classic model could only describe the propagation process on small-scale social networks because they did not consider the characteristics of social network topology [7]. In addition, scholars further studied the influence of the complex topology of social networks on the 
dynamics of rumor propagation [8]. Zanette et al. first applied the theory of complex networks to the study of rumor propagation and proposed the rumor propagation model on static and dynamic small-world networks [9]. Moreno et al. used the Monte Carlo simulation method to study the random MK model on scale-free networks [10]. Based on the traditional rumor propagation model, many scholars used different methods to study the rumor propagation mechanism [11], thus developing many new rumor propagation models, such as SIS (Susceptible-Infected-Susceptible) model [12], SIR (Susceptible-InfectedRemoved) model [13], SEIR (Susceptible-Exposed-Infected-Removed) model [14], SIHR (Susceptible-Infected-Hibernator-Removed) model [15], ILSR (Ignorant-Lurker-SpreaderRemoval) model [16], IWSR (Ignorant-Wiseman-Spreader-Stifler) model [17], and SCIR (Susceptible-Infective-Counterattack-Refractory) model [18], etc.

Some scholars have found that the activity rate of individuals will also affect the process of rumor propagation. An individual's activity rate represents the individual's willingness to spread rumors. Individuals with a higher rate of activity are usually more active in spreading rumors. Liu et al. considered the influence of individual activity rates on the spread of rumors and obtained a new critical curve consisting of pairs of critical activity rate and infection rate [19]. Huo et al. believed that the activity rate of the individuals in complex networks would affect the process of rumor propagation [20].

In reality, the negative impact of the large-scale spread of rumors has attracted official attention. The government began to formulate some measures to refute the rumors, called the rumor refutation mechanism. Some scholars discovered that the rumor refutation mechanism could effectively control rumor propagation. Zhao et al. described the dynamic process of rumor propagation by accounting for the refutation mechanism in homogeneous social networks [21]. Zhang et al. developed a dynamic eight-state ICSAR rumor propagation model considering the official rumor refutation mechanism [22]. Zhang et al. introduced a new two-stage rumor propagation and refutation model with time effect for online social networks [23]. Einwiller and Kamins considered the moderating effect of rumors-targeted identity on rumor impact and refutation effectiveness [24]. Jia et al. studied a rumor propagation model with regime switching considering colored noise and white noise and calculated a threshold Rs that guarantees the existence of rumor demise and stationary distribution [25].

In addition, the propagation of rumors is influenced not only by the global social environment but also the local environment. For instance, in terms of the global impact of the spread of rumors, Zhao et al. studied the SIHR rumor propagation model considering the forgetting and remembering mechanisms in a homogeneous network [26]. Dong et al. established an SEIR rumor propagation model to describe online social networks with different total user numbers and user deactivation rates [27]. Zhu studied the comprehensive influence of psychological factors, propagation delay, network topology, and control strategies on the spread of rumors on online social networks [28]. Li et al. considered the influence of government punishment on SIS and SIR rumor propagation models [29]. Li et al. proposed a model considering the symmetry and complexity of the social strengthening effect [30]. In terms of the local influence of the spread of rumors, Wang et al. studied the influence of differences in network individual identification capabilities on rumor propagation [31]. Xu et al. introduced an enhanced mechanism to describe the individual's willingness to spread rumors [32]. Cheng et al. consider the influence of psychological factors such as the weakening of personal interest and the cumulative effect of memory on rumor propagation [33]. Hu et al. considered and discussed different attitudes of individuals to the spread of rumors and established a sensitive-hesitant-affected-resistance (SHAR) model [34]. Chen et al. divided the population into two categories: radical and stable and proposed the SEIsIrR model [35].

From the above literature research, it can be seen that the rumor refutation mechanism and individual activity rates have a certain impact on rumor propagation. In the previous literature, the impact of the rumor refutation mechanism was not analyzed into two aspects: global and local. Therefore, on the basis of the classic ISR rumor propagation model, this 
paper considers the global and local rumor refutation mechanism and individual activity rates, establishes the mean-field equation, deduces the rumor propagation model, calculates the threshold of rumor propagation, and conducts simulation analysis on a heterogeneous network. The arrangement of this paper is as follows. Section 2 introduces the improved rumor propagation model in detail. In Section 3, we use the mean-field equation to derive the corresponding equations of the model. In Section 4, the numerical study is carried out to verify the theoretical analysis. Section 5 presents the conclusions.

\section{The Dynamic Rumor Propagation Model}

In this paper, we considered two aspects that may affect the spread of rumors: the global and the local influences. On the one hand, in the process of rumor propagation, the government will launch corresponding refutation strategies based on the scale and severity of the spread of rumors. In other words, the government will determine the strength of rumor refutation according to the severity of the rumor influence to restrain the spread of rumors. Under the global influence of the rumor refutation mechanism, the public opinion atmosphere of the whole society began to change, thus changing people's attitude towards the rumor and lowering the threshold of rumor propagation. On the other hand, NGOs and some people will also spontaneously refute rumors and have an impact on individuals around them. The rumor refutation mechanism of NGOs also affects people's attitude towards rumors and reduces people's willingness to spread rumors. In the heterogeneous networks, we regard individuals as nodes.

In the traditional rumor propagation ISR model, each node can be in one of the following three states: ignorant ( $I$, those who have never heard the rumor), spreader $(S$, those who spread the rumor), stifler ( $R$, those who have heard the rumor but not to spread), and each link represent the contact between nodes. In this paper, we also used the ISR model to describe the dynamic process of rumor propagation. Moreover, we noticed that different individuals have different activity rates. In the process of rumor propagation, there were roughly two types of individuals: active and inactive. Individuals with different activity rates will react differently when faced with rumors. These two types of people have different attitudes towards rumor propagation. Active individuals are very proactive in the rumor propagation and willing to share information or gossip with people around them. This is to say, active individuals can interact or exchange information with all individuals. Inactive individuals have a negative attitude towards rumor propagation and will not take the initiative to contact other people and spread rumors, which means that two inactive individuals may not exchange information with each other. However, as their opinions change, inactive individuals may become active, and active individuals may become inactive. We assume that active individuals and inactive individuals can transform into each other with a certain probability and introduce the activity rate to express this difference. We set $\alpha$ represents the probability of a node is in active state and $1-\alpha$ is in inactive state. Therefore, all nodes will be in one of the following six states: active ignorant $\left(I^{a}\right)$, inactive ignorant $\left(I^{d}\right)$, active spreader $\left(S^{a}\right)$, inactive spreader $\left(S^{d}\right)$ and active stifler $\left(R^{a}\right)$, inactive stifler $\left(R^{a}\right)$, in which the superscript $a$ denotes an active state and $d$ inactive state. Since stifler has no effect on the process of rumor propagation, we use $R$ instead of $R^{a}$ and $R^{d}$.

We divided all nodes into three groups according to their degree and gave the meanfield analysis to study the spread dynamics of rumor propagation. The number of nodes in each group $k$ is denoted by $N(k)$. Then, we use $I(k, t), S(k, t), R(k, t)$ to represent the expected number of nodes with degree $k$ at time $t$ in ignorant, spreader and stifler state, and $\rho^{I}(k, t), \rho^{S}(k, t), \rho^{R}(k, t)$ as their density respectively. Apparently, $\rho^{S}(k, t)=S(k, t) / N(k)$, $\rho^{R}(k, t)=R(k, t) / N(k), \rho^{I}(k, t)=I(k, t) / N(k)$ and $\rho^{I}(k, t)+\rho^{S}(k, t)+\rho^{R}(k, t)=1$.

In our model, the global rumor refutation mechanism is dominated by the government and has an impact on all people in the whole society. Time delay is not considered in this model. We assume that once the rumors begin to spread, the government will take measures to refute rumors according to the situation. Moreover, the more people infected, 
the stronger the government's efforts to refute rumors. After activating the rumor refutation mechanism, the social environment of rumor propagation has changed, which is called global influence, denoted by $\xi_{g}$. The local rumor refutation mechanism is dominated by the NGOs. It affects a small range of people through the contact among individuals. Under the influence of the local rumor refutation mechanism, the individual's willingness to spread rumors will be reduced accordingly. We call it local influence, which is represented by $\xi_{l}$.

When the rumor spreads with probability $\lambda$, affected by the global and local influence of rumor refutation mechanism, the spread probability when an active ignorant node $i$ contacts the surrounding $g(g \leq k)$ spreader nodes at time $t$ who has heard the rumor in the time interval $[t, t+\Delta t]$ is $\lambda\left(1-\xi_{g}\right)\left(1-\xi_{l}\right)$. Therefore, the probability of an active ignorant $i$ becoming an active spreader when there are $n$ spreaders around is

$$
P_{I \rightarrow S}^{a}=1-\left[1-\lambda\left(1-\xi_{g}\right)\left(1-\xi_{l}\right)\right]^{n}
$$

In the process of rumor propagation, active nodes play a relatively major role. Since active and inactive nodes can be transformed into each other with probability $\alpha$ and $1-\alpha$. For inactive nodes, the probability of transformation from ignorant to spreaders will be affected by $\alpha$. To simplify the calculation, we consider the influence of $\alpha$ as the influence of the whole network. Moreover, the probability of an inactive ignorant node $i$ becoming an inactive spreader when there are $m$ active spreaders around is

$$
P_{I \rightarrow S}^{d}=1-\left[1-\lambda \alpha\left(1-\xi_{g}\right)\left(1-\xi_{l}\right)\right]^{m}
$$

where $\xi_{g}=f\left(\rho_{\text {global }}^{S}(t)\right)$ is global influence of the rumor refutation mechanism, which means that the government decides the strength of rumor refutation based on the proportion of infected nodes in the whole network. Moreover, we assume the global influence of the rumor refutation mechanism as a set of linear relationships, that is, the function of the government's rumor refutation intensity and the density of the rumor spreaders. In addition, considering that inactive nodes will only be affected by active spreaders, we use $g$ instead of $m$ and $n$ to represent the number of spreaders around the ignorant node $i$. Therefore, $\xi_{g}=f\left(\rho_{\text {global }}^{S}(t)\right)=b \rho^{S}(t)$, where $b \in(0,1)$ is the strength of the government to refute rumors and $\rho^{S}(t)$ is taken to be representative of the density of spreaders in whole network. Similarity, $\xi_{l}=h\left(\rho_{\text {local }}^{S}(t)\right)=\gamma g / k$ is taken to be representative of the local influence of rumor refutation mechanism, where $\gamma \in(0,1)$ is the coefficient of NGOs rumor refutation mechanism and $k$ is the total number of its neighbors.

The contacts rules between the active and inactive individuals are as follows:

- In the process of rumor propagation, affected by the global and local influence of rumor refutation mechanism, when an active spreader $\left(S^{a}\right)$ contacts an active or inactive ignorant $\left(I^{a}\right.$ or $\left.I^{d}\right)$, the active ignorant becomes an active spreader with probability $P_{I^{a} \rightarrow S^{a}}^{a}$ or $P_{I^{d} \rightarrow S^{d}}^{a}$, respectively. Moreover, we have $P_{I^{a} \rightarrow S^{a}}^{a}=\omega P_{I^{d} \rightarrow S^{d}}^{a}$ where $0 \leq \omega \leq 1$. To simplify the calculation, we let $\omega=1$, thus $P_{I^{a} \rightarrow S^{a}}^{a}=P_{I^{d} \rightarrow S^{d}}^{a}$. We use $P_{I \rightarrow S}^{a}$ instead of $P_{I^{a} \rightarrow S^{a}}^{a}$ and $P_{I^{d} \rightarrow S^{d}}^{a}$. The inactive ignorant becomes an inactive spreader with probability $P_{I \rightarrow S}^{d}$;

- For some reasons, the active and inactive individuals can transform into each other with a certain probability. The probability that the active individual becomes the inactive state is $\alpha$, otherwise $1-\alpha$. The active ignorant can be infected by active and inactive spreader, but the inactive ignorant only can be infected by active spreader;

- When the spreader contacts another spreader or stifler, the spreader loses the motivation to spread the rumors and becomes a stifler with probability $\beta$;

- Due to the forgetting mechanism, active or inactive spreaders change their states and become stiflers spontaneously with probability $\delta$.

The reaction process can be schematically represented by 
1. Ignorant state change

$$
\begin{aligned}
& I^{a}+S^{a} \stackrel{P_{I \rightarrow}^{a}}{\rightarrow} S^{a}+S^{a} \\
& I^{a}+S^{d} \stackrel{P_{I \rightarrow}^{a}}{\rightarrow} S^{a}+S^{d} \\
& I^{d}+S^{a} \stackrel{P_{I \rightarrow S^{d}} S}{S^{d}} S^{a}+S^{a}
\end{aligned}
$$

2. Spreader state change

$$
\begin{aligned}
& S^{a}+S^{a} \stackrel{\beta}{\rightarrow} R+S^{a} \\
& S^{a}+S^{d} \stackrel{\beta}{\rightarrow} R+S^{d} \\
& S^{a}+R \stackrel{\beta}{\rightarrow} R+R \\
& S^{d}+R \stackrel{\beta}{\rightarrow} R+R
\end{aligned}
$$

3. Spontaneously stop spreading rumors

$$
\begin{aligned}
& S^{a} \stackrel{\delta}{\rightarrow} R \\
& S^{d} \stackrel{\delta}{\rightarrow} R
\end{aligned}
$$

According to the above description, we can obtain the rumor propagation process as shown in Figure 1.

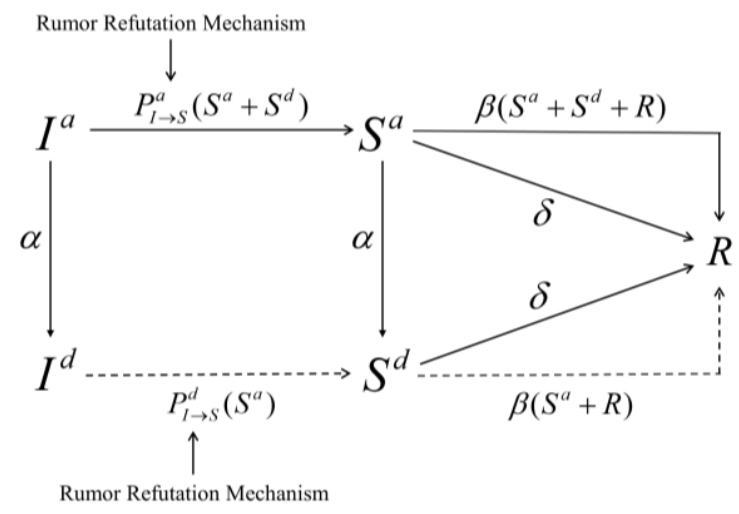

Figure 1. The dynamic process of rumor propagation.

Based on the above assumptions, we establish an improved ISR model that considers the rumor refutation mechanism and activity rates. In Section 3, we will derive the meanfield equation for the rumor propagation model and calculate the critical threshold.

\section{Mean-Field Equations of Spreading Dynamics}

In this section, we consider the mean-field equations of the above spreading dynamic model on the heterogeneous networks. According to the improved model proposed in Section 2 , let $\phi\left(k^{\prime}, t\right)$ be the probability that a node with degree $k$ randomly selected edge points to a spreader, thus we have

$$
\phi\left(k^{\prime}, t\right)=\frac{\sum_{k^{\prime}} k^{\prime} P\left(k^{\prime}\right) \rho^{S}\left(k^{\prime}, t\right)}{\langle k\rangle}
$$

where $P\left(k^{\prime}\right)$ is the degree distribution of the network, $\langle k\rangle=\sum_{k} k P(k)$ is the average degree and $\rho^{S}\left(k^{\prime}, t\right)$ is the density of spreaders with degree $k^{\prime}$ at time $t$.

Generally, active ignorant individuals can be infected by any infected individual, but inactive ignorant individuals can only be infected by active infected individuals. Next, we will divide the discussion into two cases. 
The probability that a node with a degree $k$ has $g$ spreading neighbors is given by

$$
\theta(k, g)=C_{k}^{g} \phi\left(k^{\prime}, t\right)^{g}\left(1-\phi\left(k^{\prime}, t\right)\right)^{k-g}=\frac{g !}{k !(g-k) !} \phi\left(k^{\prime}, t\right)^{g}\left(1-\phi\left(k^{\prime}, t\right)\right)^{k-g}
$$

For further derivation, we let $\phi=\phi\left(k^{\prime}, t\right)$ and $\theta=\theta(k, g)$. Combined with Equation (1), the probability that an active ignorant keeps its state unchanged for arbitrary $g$ at time $t$ is

$$
P_{I I}^{a}(k, t)=\sum_{g=0}^{k}\left(1-P_{I \rightarrow S}^{a}\right) \theta=1-\sum_{g=0}^{k}\left[1-\lambda\left(1-b \rho^{S}(t)\right)(1-\gamma g / k) \Delta t\right]^{g} \theta
$$

By employing L’Hôpital's rule, we obtain

$$
\begin{aligned}
& \lim _{\Delta t \rightarrow 0} \frac{1}{\Delta t}\left[1-\sum_{g=0}^{k}\left(1-\lambda\left(1-b \rho^{S}(t)\right)(1-\gamma g / k) \Delta t\right)^{g} \theta\right] \\
& =1-\lim _{\Delta t \rightarrow 0} \sum_{g=0}^{k} g \theta\left(1-\lambda \Delta t\left(1-b \rho^{S}(t)\right)(1-\gamma g / k)\right)^{g-1} \lambda \Delta t\left(1-b \rho^{S}(t)\right)(1-\gamma g / k) \\
& =1-\lambda \Delta t\left(1-b \rho^{S}(t)\right) \sum_{g=0}^{k} g \theta(1-\gamma g / k)
\end{aligned}
$$

Thus, we can obtain

$$
P_{I I}^{a}(k, t)=\sum_{g=0}^{k}\left(1-P_{I \rightarrow S}^{d}\right) \theta=1-\lambda \Delta t\left(1-b \rho^{S}(t)\right) \sum_{g=0}^{k} g \theta(1-\gamma g / k)
$$

Similarity, the probability that an inactive ignorant keeps its state unchanged for arbitrary $g$ at time $t$ is

$$
P_{I I}^{d}(k, t)=1-\lambda \alpha \Delta t\left(1-b \rho^{S}(t)\right) \sum_{g=0}^{k} g \theta(1-\gamma g / k)
$$

Based on above analysis, the probability of an ignorant node with degree $k$ to stay in ignorant state at time $t$ is

$$
\begin{aligned}
P_{I I}(k, t) & =\alpha P_{I I}^{a}+(1-\alpha) P_{I I}^{d} \\
& =\alpha\left[1-\lambda\left(1-b \rho^{S}(t)\right) \sum_{g=0}^{k} g \theta(1-\gamma g / k)\right]+(1-\alpha)\left[1-\lambda \alpha\left(1-b \rho^{S}(t)\right) \sum_{g=0}^{k} g \theta(1-\gamma g / k)\right] \\
& =1-\left(2 \alpha-\alpha^{2}\right) \lambda \Delta t\left(1-b \rho^{S}(t)\right) \sum_{g=0}^{k} g \theta(1-\gamma g / k)
\end{aligned}
$$

The discrete-time rumor propagation process with some simplification can be described as the following equation according to Equation (3)

$$
\begin{aligned}
\rho^{I}(k, t+\Delta t) & =\rho^{I}(k, t)-\left(1-P_{I I}\right) \rho^{I}(k, t) \\
& =\rho^{I}(k, t)-\left(2 \alpha-\alpha^{2}\right) \lambda \Delta t \rho^{I}(k, t)\left(1-b \rho^{S}(t)\right) \sum_{g=0}^{k} g \theta(1-\gamma g / k)
\end{aligned}
$$

Dividing both sides of Equation (4) by $\Delta t$ and letting $\Delta t \rightarrow 0$, Equation (4) can be written as a continuous-time equation

$$
\frac{d \rho^{I}(k, t)}{d t}=-\left(2 \alpha-\alpha^{2}\right) \lambda \rho^{I}(k, t)\left(1-b \rho^{S}(t)\right) \sum_{g} g \theta(1-\gamma g / k)
$$


Then, we calculate the value of $\sum_{g} \lambda g \theta(1-\gamma g / k)$

$$
\lambda \sum_{g} g \theta(1-\gamma g / k)=\lambda \sum_{g}\left(g \theta-\gamma g^{2} / k \theta\right)=\lambda E(g)-\lambda \gamma E\left(g^{2} / k\right)
$$

where $E(g)=k \phi, E\left(g^{2} / k\right)=k \phi^{2}+\phi(1-\phi)$. Similarity, we can obtain

$$
\begin{gathered}
\frac{d \rho^{S}(k, t)}{d t}=\left(2 \alpha-\alpha^{2}\right) \lambda(1-b) \rho^{I}(k, t)\left[E(g)-\gamma E\left(g^{2} / k\right)\right] \\
-\left(2 \alpha-\alpha^{2}\right) \rho^{S}(k, t) \beta E^{\prime}(g)-\delta \rho^{S}(k, t) \\
\frac{d \rho^{R}(k, t)}{d t}=\left(2 \alpha-\alpha^{2}\right) \rho^{S}(k, t) \beta E^{\prime}(g)+\delta \rho^{S}(k, t)
\end{gathered}
$$

where $E^{\prime}(g)=k \phi^{\prime}, E^{\prime}\left(g^{2} / k\right)=k \phi^{\prime 2}+\phi^{\prime}\left(1-\phi^{\prime}\right)$ and

$$
\varphi\left(k^{\prime}, t\right)=\sum_{k^{\prime}} \frac{k^{\prime} P\left(k^{\prime}\right)\left(\rho^{S}\left(k^{\prime}, t\right)+\rho^{R}\left(k^{\prime}, t\right)\right)}{\langle k\rangle}=1-\sum_{k^{\prime}} \frac{k^{\prime} P\left(k^{\prime}\right) \rho^{I}\left(k^{\prime}, t\right)}{\langle k\rangle}
$$

In order to further calculation, we set an intermediate variable $\Omega(t)$ as follows:

$$
\Omega(t)=\int_{0}^{t} \phi d t=\int_{0}^{t} \frac{\sum_{k^{\prime}} k^{\prime} P\left(k^{\prime}\right) \rho^{S}\left(k^{\prime}, t\right)}{\sum_{k^{\prime}} k^{\prime} P\left(k^{\prime}\right)} d t=\int_{0}^{t}\left\langle\left\langle\rho^{S}\left(k^{\prime}, t\right)\right\rangle\right\rangle d t
$$

In the following formula we use the following simplified symbols

$$
\int_{0}^{t} \frac{\sum_{k^{\prime}} k^{\prime} P\left(k^{\prime}\right) O(k)}{\sum_{k^{\prime}} k^{\prime} P\left(k^{\prime}\right)} d t=\int_{0}^{t}\langle\langle O(k)\rangle\rangle d t
$$

Since $E(g)=k \phi$ and $E\left(g^{2} / k\right)=k \phi^{2}+\phi(1-\phi)$, according to Equation (5), we have

$$
\begin{aligned}
\frac{d \rho^{I}(k, t)}{\rho^{I}(k, t) d t} & =-\left(2 \alpha-\alpha^{2}\right) \lambda(1-b)\left[E(g)-\gamma E\left(g^{2} / k\right)\right] \\
& =-\left(2 \alpha-\alpha^{2}\right) \lambda(1-b)\left[k \phi-\gamma\left(k \phi^{2}+\phi(1-\phi)\right)\right]
\end{aligned}
$$

Due to $\rho^{I}(k, t) \approx 0$, by removing the high order term of $\phi$, we can obtain

$$
\rho^{I}(k, t)=e^{-\lambda\left(2 \alpha-\alpha^{2}\right)(1-b)(k-\gamma) \Omega(t)}
$$

Combining Equations (5) and (8), we can derive

$$
\begin{aligned}
\frac{d \Omega(t)}{d t} & =1-\left\langle\left\langle e^{-\lambda\left(2 \alpha-\alpha^{2}\right)(1-b)(k-\gamma) \Omega(t)}\right\rangle\right\rangle \\
& -\left(2 \alpha-\alpha^{2}\right) \beta \int_{0}^{t}\left[1-\left\langle\left\langle e^{-\lambda\left(2 \alpha-\alpha^{2}\right)(1-b)(k-\gamma) \Omega(t)}\right\rangle\right\rangle\right]\left\langle\left\langle k \rho^{S}\left(k^{\prime}, t\right)\right\rangle\right\rangle d t-\delta \Omega(t)
\end{aligned}
$$

In order not to lose generality, we assume the initial distribution of ignorant is $\rho^{S}(k, t) \approx 1$. In the limit $t \rightarrow \infty$ we have $d \Omega(t) / d t=0, \Omega(\infty)=\lim _{t \rightarrow \infty} \Omega(t)$, then we can then obtain

$$
\begin{aligned}
\Omega(t) & =\left(\left(2 \alpha-\alpha^{2}\right) \beta k+\delta\right)^{-1}\left(1-\left\langle\left\langle e^{-\lambda\left(2 \alpha-\alpha^{2}\right)(1-b)(k-\gamma) \Omega(t)}\right\rangle\right\rangle\right. \\
& \left.-\left(2 \alpha-\alpha^{2}\right) \beta \int_{0}^{t}\left\langle\left\langle e^{-\lambda\left(2 \alpha-\alpha^{2}\right)(1-b)(k-\gamma) \Omega(t)}\right\rangle\right\rangle\left\langle\left\langle k \rho^{S}\left(k^{\prime}, t\right)\right\rangle\right\rangle d t\right) \\
& =f(\Omega)
\end{aligned}
$$


There is a zero solution $\Omega=0$ of Equation (9), and the necessary condition for the existence of non-zero solution of Equation (9) is

$$
\left.\frac{d f(\Omega)}{d \Omega}\right|_{\Omega=0} \geq 1
$$

which means the spreading threshold of ISR dynamic is

$$
\lambda_{c}=\frac{\delta\langle k\rangle}{\left(2 \alpha-\alpha^{2}\right)(1-b)\left(\left\langle k^{2}\right\rangle-\gamma\langle k\rangle\right)}
$$

But we must notice that $\lambda_{c}$ is not always make sense. $\lambda_{c}$ will be greater than 1 when $\alpha<1-\sqrt{1-\delta\langle k\rangle /\left(\left\langle k^{2}\right\rangle-\gamma\langle k\rangle\right)}$. This means that when the activity rate of a node is below a certain threshold, the propagation scale will be relatively small regardless of the spread rate. We can also draw the threshold of activity rate and the strength of the global rumor refutation

$$
\begin{aligned}
& \alpha_{c}=1-\sqrt{1-\frac{\delta\langle k\rangle}{\lambda(1-b)\left(\left\langle k^{2}\right\rangle-\gamma\langle k\rangle\right)}} \\
& b_{c}=1-\frac{\delta\langle k\rangle}{\lambda\left(2 \alpha-\alpha^{2}\right)\left(\left\langle k^{2}\right\rangle-\gamma\langle k\rangle\right)}
\end{aligned}
$$

From Equation (11), it can be seen that when the strength of government refutation is small, that is, when $b<b_{c}$, the rumors cannot be controlled. Only when the strength of rumor refutation is greater than $b_{c}$, can it affect the threshold of rumor propagation.

Similarity the threshold of local influence is

$$
\gamma_{c}=\frac{\left\langle k^{2}\right\rangle}{\langle k\rangle}-\frac{\delta}{\lambda\left(2 \alpha-\alpha^{2}\right)\left(\left\langle k^{2}\right\rangle-\gamma\langle k\rangle\right)}
$$

From Equation (12), we can conclude that the rumor propagation threshold $\lambda_{c}$ is related to the individual activity rate $\alpha$, the global influence of the rumor refutation mechanism $b$ and the local influence of the rumor refutation mechanism $\gamma$. Since $\rho^{S}(\infty)=0$, we obtain the final scale of rumor as follows:

$$
R(\infty)=1-\sum_{k} P(k) \rho^{I}(\infty)=\sum_{k} P(k)\left(1-e^{-\lambda\left(2 \alpha-\alpha^{2}\right)(1-b)(k-\gamma) \Omega(\infty)}\right)
$$

According to equation Equation (10), we can infer that the critical rumors propagation threshold is affected by the strength of rumor refutation $b$, the strength of spontaneous refutation $\gamma$ and the individual activity rate $\alpha$. When $b=0, \gamma=0$ and $\alpha=1$, that is, regardless of the global and local influence of rumor refutation and the nodes in the whole network are active, the spreading threshold is $\lambda_{c}=\delta\langle k\rangle /\left\langle k^{2}\right\rangle$, the model is a classical rumor propagation ISR model [11]. In particular, when $b=0$ and $\gamma=0$, it means that the government and NGOs do not refute rumors. At this time, only the influence of activity rate on the spread of rumors is considered [19].

\section{Numerical Simulations}

In this section, we will conduct numerical simulations to verify the theoretical predictions in the previous section in a heterogeneous network. We consider the global and local influence of rumor refutation mechanisms on the rumor propagation process on a single-layer network. Assume that the network is a single-layer social network on which all information is propagated. However, the model still has some shortcomings. For example, we assume that the rumor refutation mechanism only affects the transfer process from ignorant to spreader, and the change process of individual state is ignorant-spreadercontainer. But in reality, the change of individual status may be unpredictable, and the rumor refutation information may also make the ignorant directly transform into the stifler. 
To simplify the model, we performed simulations on a scale-free network based on the existing assumptions.

During the numerical simulation, the scale-free network node is $N=10^{6}$, the average degree is $\langle k\rangle=1.62$ and the secondary moment $\left\langle k^{2}\right\rangle=7.93$. The initial spreader is randomly selected to start propagation rumors during the numerical simulation. In order to reduce the error brought by random spreaders, each of the following simulation results is obtained by averaging over 50 runs. In the following, we will discuss the impact of individual activity rates and the global and local effects of the rumor refutation mechanism on rumor propagation process, as well as the final rumor propagation threshold. We will divide into three parts and conduct numerical simulation analysis. Moreover, in the following simulation, we use the proportion of people who are ultimately in $R$ state to represent the final scale of the rumor propagation.

\subsection{Influence of Activity Rates: The Relationship between $R, \lambda$ and $\alpha$ with Fixing the Value of $\gamma, b$}

Figure 2 is a heat map composed of different rumor propagation rates and node activity rates, which shows the final size of rumor propagation. In the above figure, $\alpha$ represents the activity rate of the individual. The larger the value of $\alpha$, the more active the node spread rumors. There are three lines in the figure. The solid white line corresponds to the relationship of the critical propagation rate and the activity rate to different nodes, when less than the curve, the rumor propagation will be limited within a certain range. Moreover, the horizontal dotted line corresponds to $\alpha=0.125$, when $\alpha$ is lower than this value, the final rumor size $R$ will be very small. The vertical dotted line corresponds to $\lambda=0.065$. That is to say, when $\lambda$ is lower than this value, rumors cannot be spread.

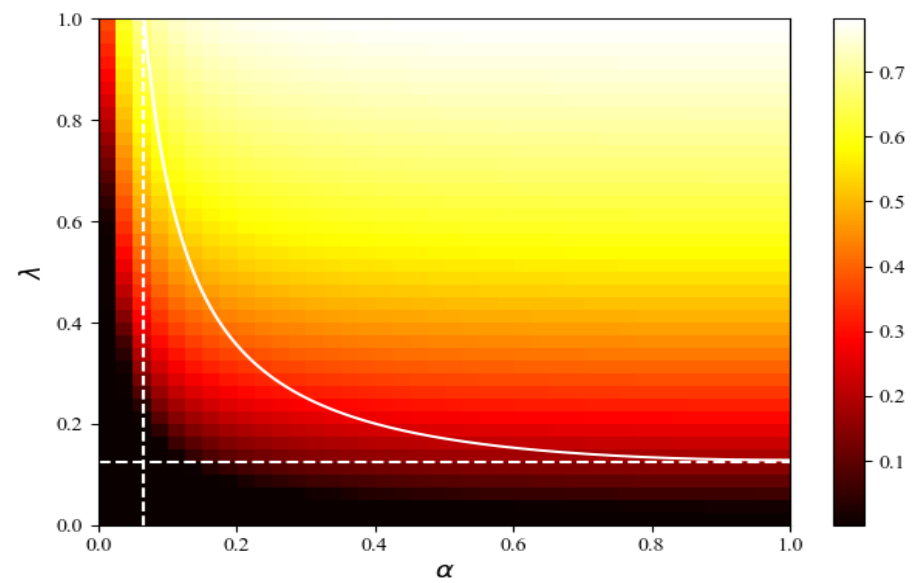

Figure 2. As shown in the figure, in the case of $\gamma=0.3$ and $b=0.5$, the relationship between the final size $R$ of the propagation dynamics and the different $\lambda$ and $\alpha$, where in the shade of the color represents the size of the propagation scale. (For interpretation of the references to color in this figure legend, the reader is referred to the web version of this article).

Figures 3 and 4 show the dynamic change of the final threshold of rumor propagation with respect to $\alpha$ and $\lambda$. Figure 3 shows the relationship between the activity rate and the final size of the rumor $R$. Moreover, it shows that the critical threshold of spread rate is highly relevant to $\alpha$. We can conclude that the final size $R$ increases with the increase of $\alpha$, and the larger the value of $\lambda$, the faster the increased speed. It means that the spread rate larger, the faster the rumor propagation, and the larger the final size of the rumor is. Therefore, controlling the scale of rumor propagation requires not only reducing the spread rate but also the activity rate of individuals. Moreover, it can be seen from Figure 4 , in the initial stage of the propagation of rumors, when $\lambda$ is very small, no matter how active the node is, the final size of the rumor $R$ is very small, which means that the rumor cannot be spread on a large scale. When the activity rate is low $(\alpha=0.025)$, only a large spread rate will cause the large-scale spread of rumors. However, as the activity rate increases, a 
smaller spread rate will also cause a large-scale spread, and the final size of the rumor will increase with the increase of $\alpha$. This means that the activity rate of nodes has accelerated the spread of rumors to a certain extent, making the final size of rumors larger.

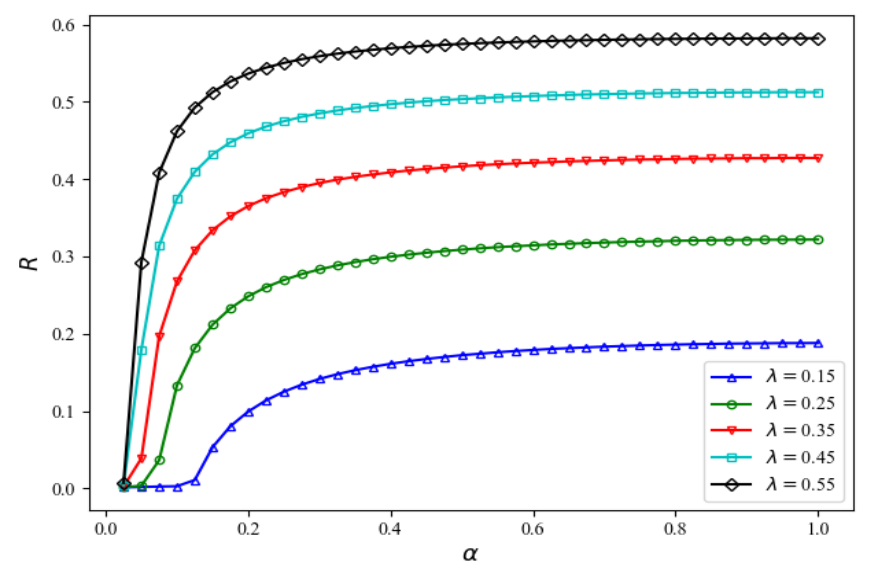

Figure 3. (Color online) The relationship between the final size $R$ and the proportion of activities $\alpha$.

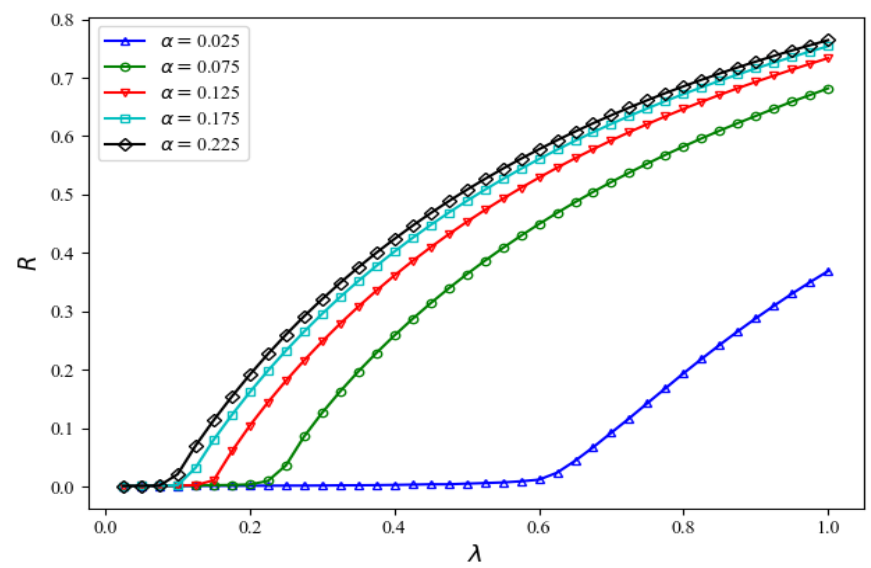

Figure 4. (Color online) The relationship between the final size $R$ and the spreading rate $\lambda$.

Figures 5 and 6 represent the number of nodes infected with this rumor and stop spreading this rumor as time changes. From Figure 5, it can be seen that when the activity rate of nodes $\alpha$ is low, its peak value is smaller and the duration of rumor propagation is longer. When $\gamma(\gamma=0.3)$ and $b(b=0.5)$ are fixed, with the increase of $\alpha$, the rumor will reach its peak value more quickly, and then gradually fall to 0 . As we can see from Figure 6, when the activity rate of nodes $\alpha$ is low, the number of stiflers is less, and the speed is slower. When $\gamma(\gamma=0.3)$ and $b(b=0.5)$ are fixed, the number of stiflers increases with the increase of $\alpha$, the rate of spreader becoming a stifler will be faster. When the activity rate of nodes increases, the interaction between nodes increases, thus, the speed of the rumor propagation and the density of spreaders and stiflers are also increasing. 


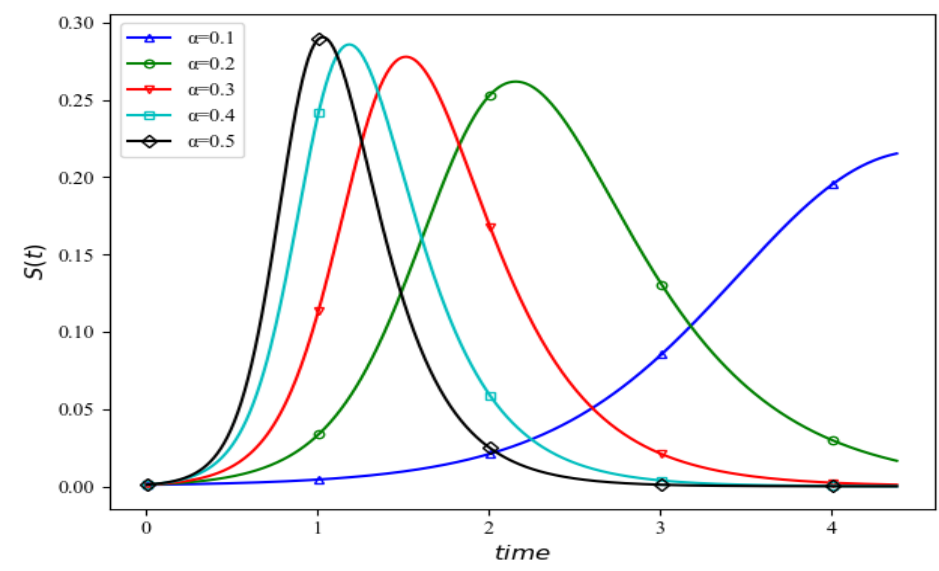

Figure 5. (Color online) Time evolution of the fraction of spreader nodes when the dynamics start with a random selected node for several values of the proportion of activities $\alpha$.

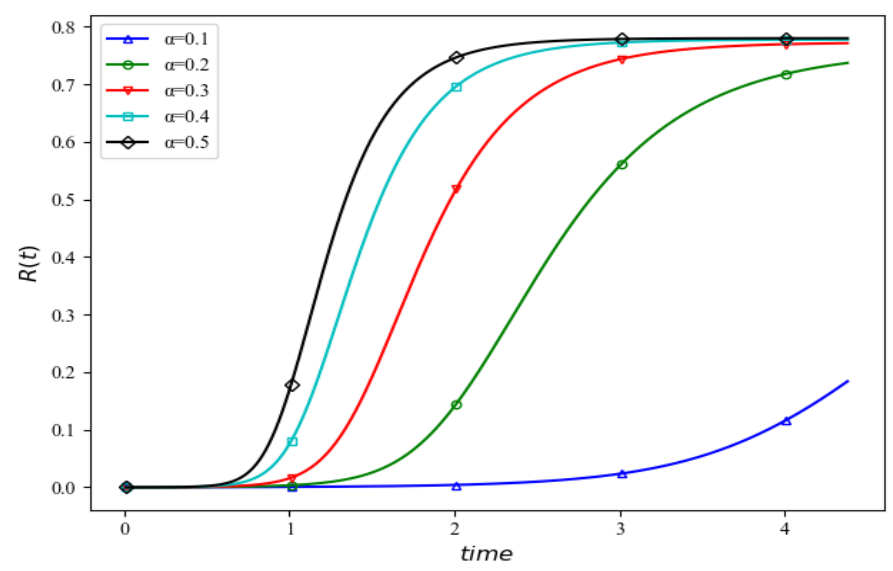

Figure 6. (Color online) Time evolution of the fraction of stifler nodes when the dynamics start with a random selected node for several values of the proportion of activities $\alpha$.

4.2. Global Influence of Refutation Mechanism: The Relationship between $R, \lambda$ and $b$ with Fixing the Value of $\gamma, \alpha$

Figure 7 is a heat map composed of different rumor propagation rates and the strength of the rumor refutation mechanism. The solid white line corresponds to the relationship of the critical propagation rate and the strength of the rumor refutation mechanism. $b$ represents the strength of rumor refutation. The higher the value of $b$, the larger the government's efforts to refute rumors, and the smaller the final rumor size $R$. In this way, rumors can be controlled, and the negative influence of rumor propagation can be reduced.

Figures 8 and 9 show the dynamic change of the final threshold of rumor propagation with respect to $b$ and $\lambda$. Figure 8 shows the relationship between the strength of rumor refutation $b$ and the final size of the rumor $R$. We can conclude that the size of $R$ decreases with the increase of $b$, and the larger the value of $\lambda$, the faster the increased speed. It means that the greater the effort to refute rumors, the smaller the final size of the rumors. Regardless of the size of $\lambda$, with the increase of $b$, the final value of $R$ will eventually become very small. It also shows that the rumor can be controlled only when the strength of rumor refutation reaches a certain threshold (i.e., $b>b_{c}$ ). Moreover, in Figure 9, we can see that as the value of $b$ increases, the size of the final rumor $R$ decreases, and there is an inverse relationship between $b$ and $R$. When $b$ is large and the spread rate is low, rumors will not spread on a large scale. The greater the government's efforts to refute rumors, the stronger the control over the spread of rumors. When the rumors are strongly refuted, the final size of the rumors becomes very small. 


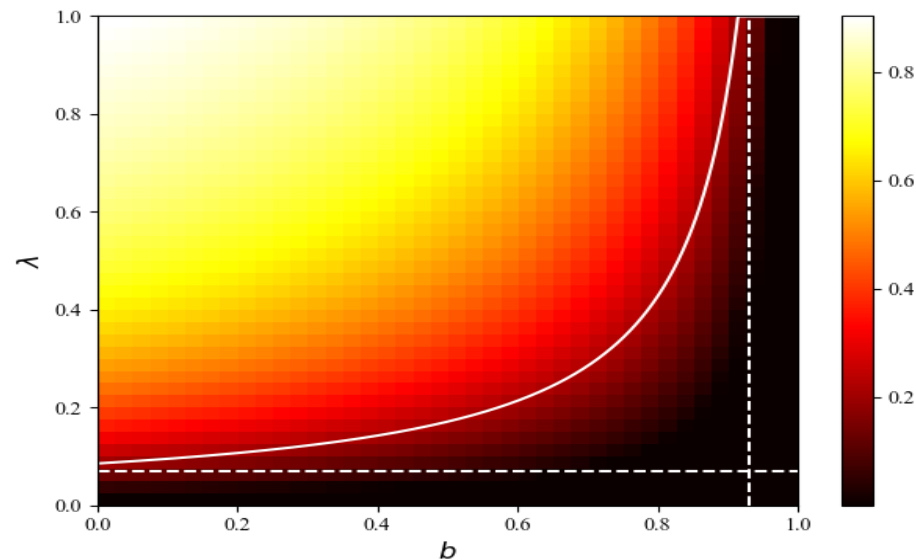

Figure 7. As shown in the figure, in the case of $\gamma=0.3$ and $\alpha=0.5$, the relationship between the final size $R$ of the propagation dynamics and the different $\lambda$ and $b$, where in the shade of the color represents the size of the propagation scale. (For interpretation of the references to color in this figure legend, the reader is referred to the web version of this article.).

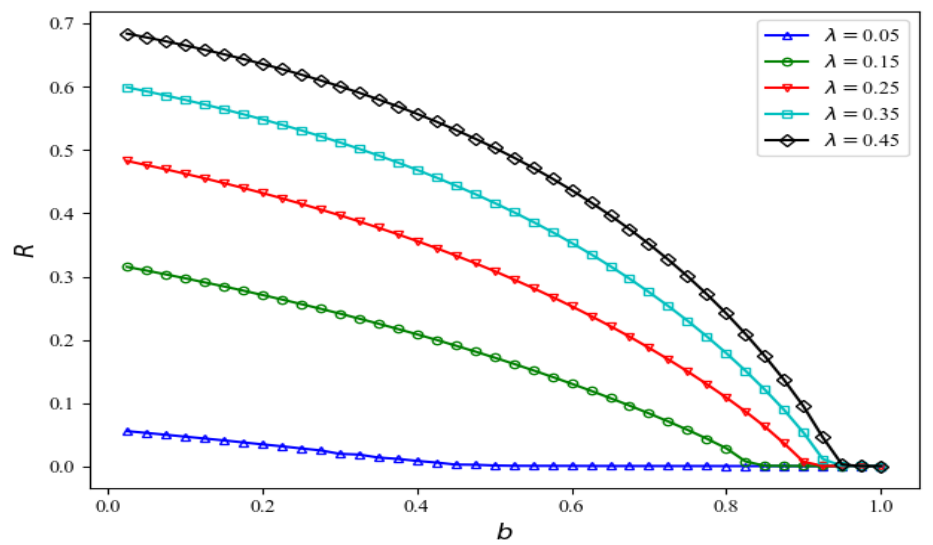

Figure 8. (Color online) The relationship between the final size $R$ and the strength of rumor refutation $b$.

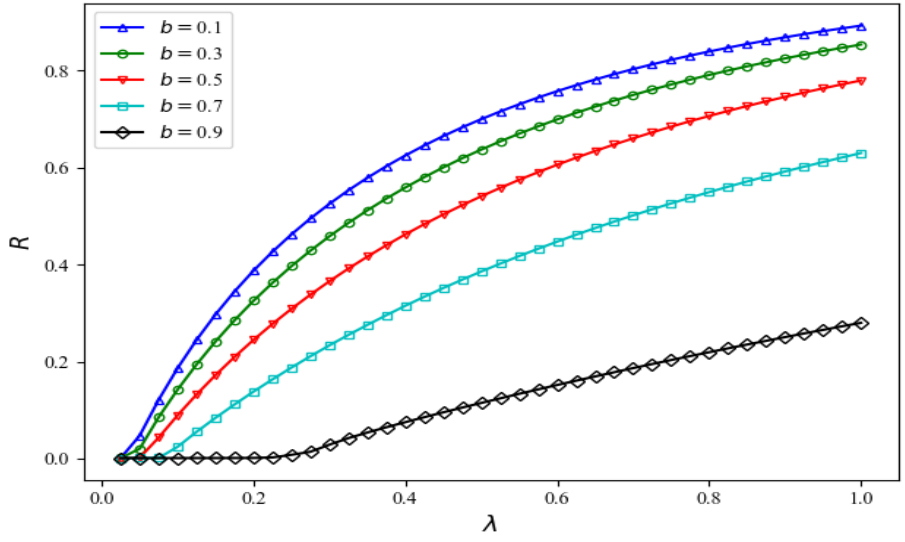

Figure 9. (Color online) The relationship between the final size $R$ and the spreading rate $\lambda$.

Figures 10 and 11 reflect the curves of spreader and stifler density over time, respectively. It can be seen in Figure 10 that when the strength to refute rumors are low, that is, the value of $b$ is small, rumors spread very quickly, and the peak value of the density of spreaders is higher. When $\alpha(\alpha=0.5)$ and $\gamma(\gamma=0.5)$ are fixed, with the increase of $b$, the rumor will reach its peak value more slowly, and then gradually fall to 0 . When $b=0.75$, the speed of rumors spreading is slow, and the peak value of the density of spreaders is 
correspondingly low. As we can see from Figure 11, the density of stiflers also decreased as the strength of rumor refutation increased. When the government's efforts to refute rumors increase, the density of spreaders and stiflers decreases at the same time, which shows that the global strength of rumor refutation has an important impact on the spread of rumors.

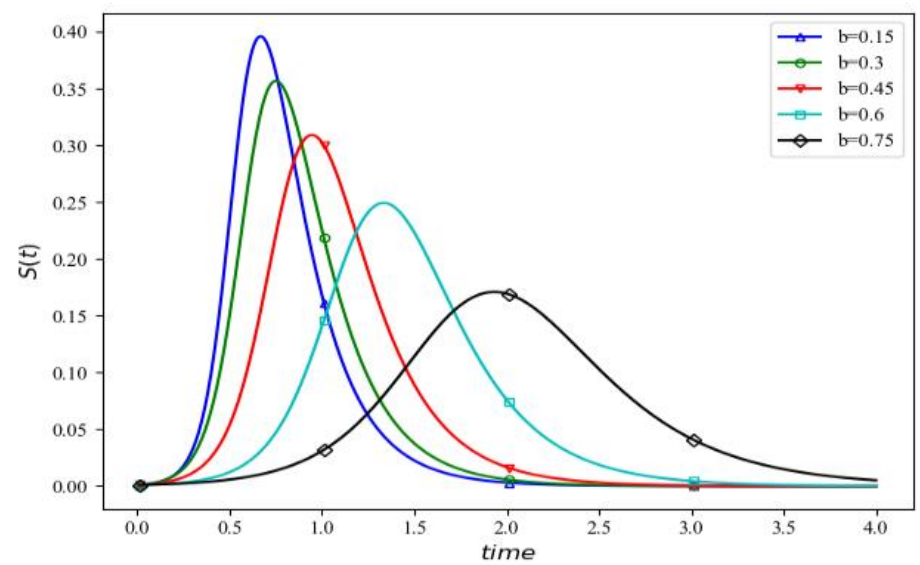

Figure 10. (Color online) Time evolution of the fraction of spreader nodes when the dynamics start with a random selected node for several values of the strength of rumor refutation $b$.

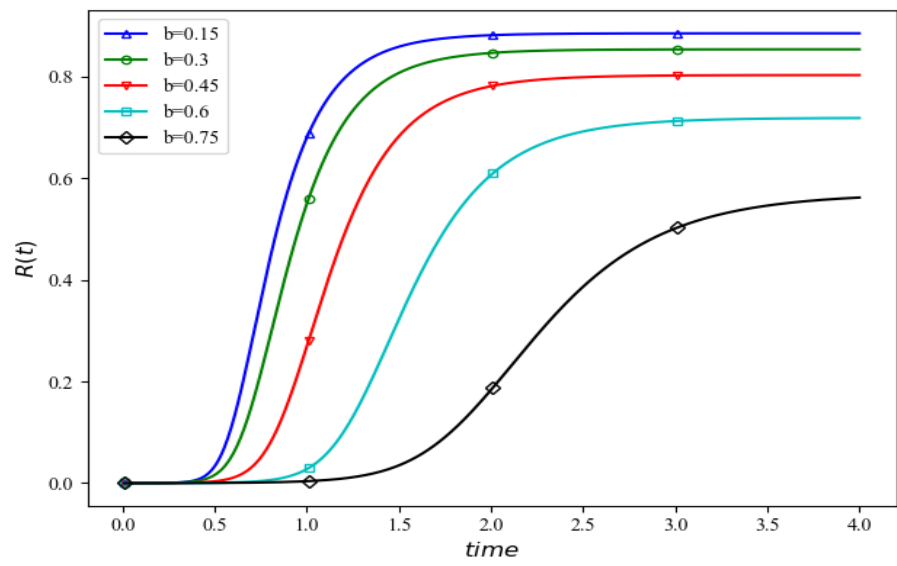

Figure 11. (Color online) Time evolution of the fraction of stiflers nodes when the dynamics start with a random selected node for several values of the strength of rumor refutation $b$.

4.3. Local Influence of Refutation Mechanism: The Relationship between $R, \lambda$ and $\gamma$ with Fixing the Value of $\alpha, b$

Figure 12 is a heat map composed of different rumor propagation rates and the strength of NGOs spontaneous rumor refutation. The higher the value of $\gamma$, the more the individual will be affected by the local rumor refutation mechanism and the lower the willingness to spread rumors.

Figures 13 and 14 show the dynamic change of the final threshold of rumor propagation with respect to $\gamma$ and $\lambda$. From Figure 13, we can see that as $\gamma$ increases, the threshold of the rumor is also slowly decreasing. Moreover, no matter how much the spread rate $\lambda$ is, the local influence of the rumor refutation mechanism can inhibit the spread of rumors to a certain extent. In particular, when $\gamma=0$, it means that people do not exchange information about refutation, thus the size of $R$ increases with the increase of $\lambda$. In Figure 14, we see that the local rumor refutation mechanism has little effect on the threshold of rumor propagation. No matter how the size of $\gamma$ changes, the final rumor size will hardly change. It shows that people's trust in the rumor refutation mechanism of NGOs is not high. Because in the highly developed modern social network today, people have rarely transmitted and exchanged information they obtained on the network to the people around them. Most of 
the channels for people to receive and transmit information are on the Internet. Therefore, the rumor refutation mechanism in the social circle, that is, the local influence of the rumor refutation mechanism, does not significantly affect the final size of the rumor propagation.

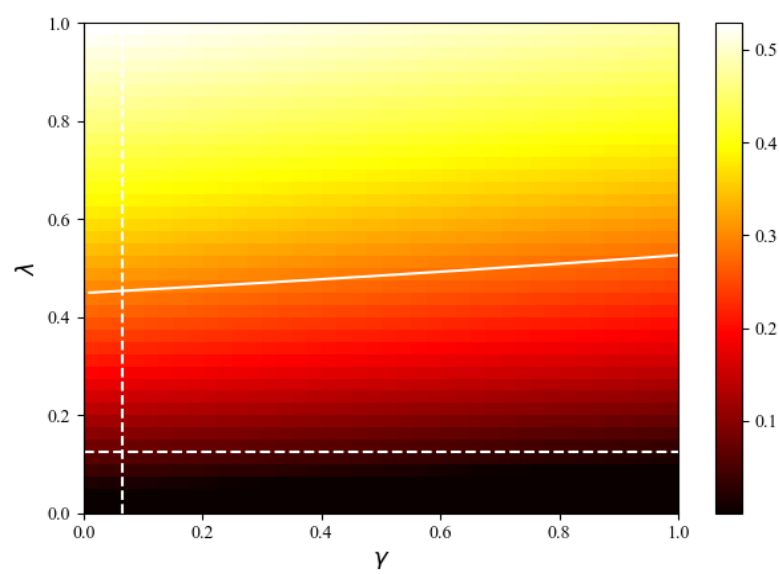

Figure 12. As shown in the figure, in the case of $b=0.7$ and $\alpha=0.7$, the relationship between the final size $R$ of the propagation dynamics and the different $\lambda$ and $\gamma$, where in the shade of the color represents the size of the propagation scale. (For interpretation of the references to color in this figure legend, the reader is referred to the web version of this article).

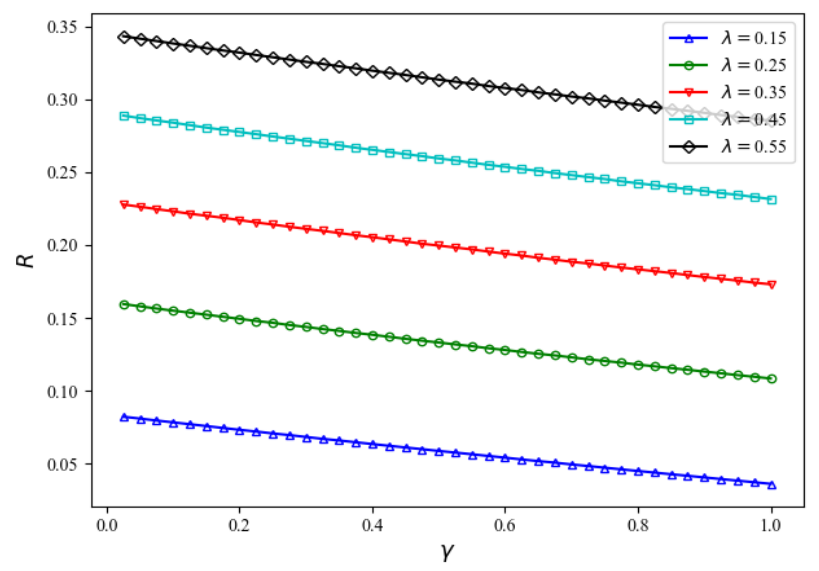

Figure 13. (Color online) The relationship between the final size $R$ and the coefficient of spontaneous rumor refutation $\gamma$.

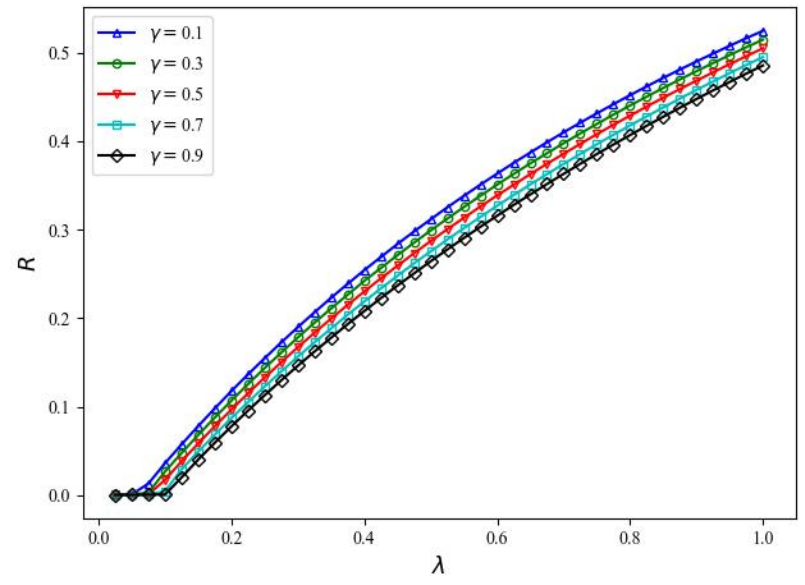

Figure 14. (Color online) The relationship between the final size $R$ and the spreading rate $\lambda$. 
Figures 15 and 16 reflect the curves of spreader and stifler density over time, respectively. As analyzed above, the local influence of the refutation mechanism between people has little effect on the spread of rumors, thus the density of spreaders and stiflers hardly changes with $\gamma$. At the same time, it also shows that relying on channels between friends to spread rumors is an unstable method because few people completely believe in the rumors raised by friends on social platforms or face-to-face communication.

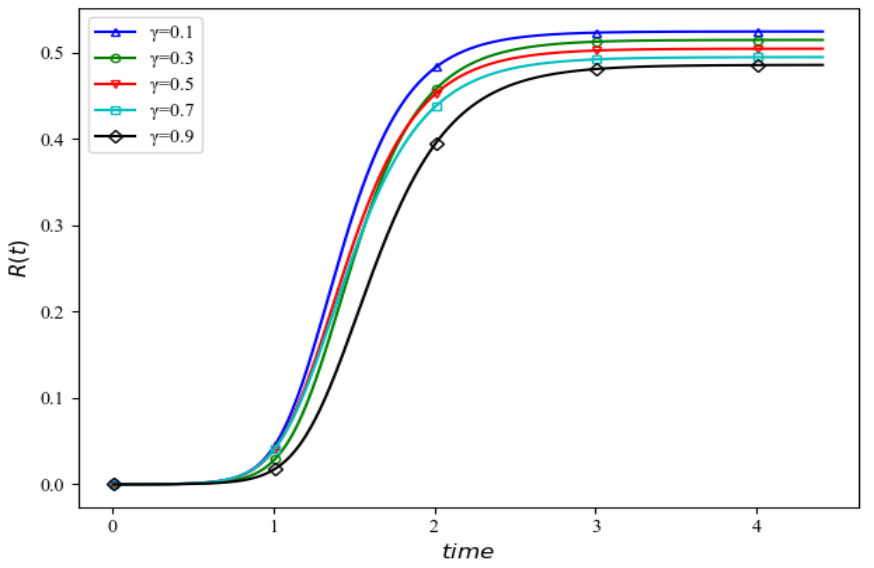

Figure 15. (Color online) Time evolution of the fraction of spreaders nodes when the dynamics start with a random selected node for several values of coefficient of spontaneous rumor refutation $\gamma$.

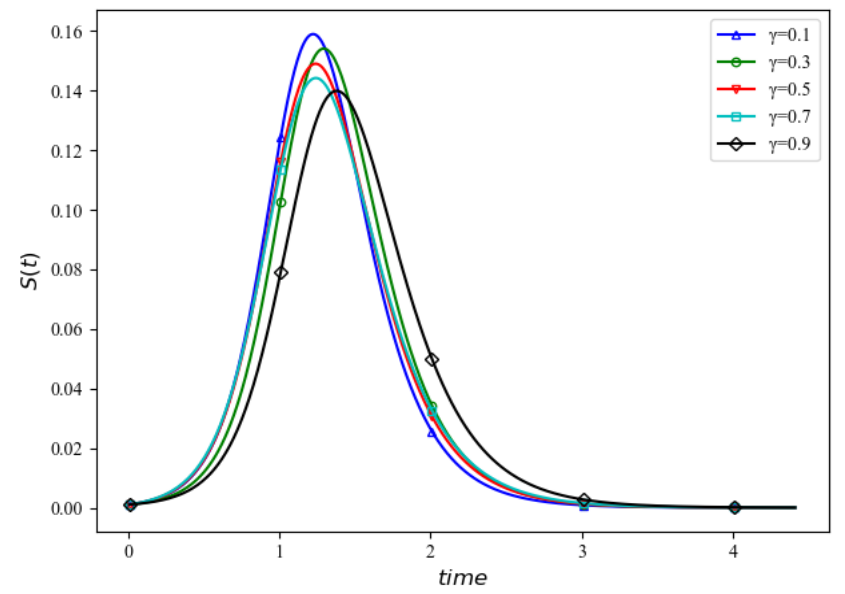

Figure 16. (Color online) Time evolution of the fraction of stiflers nodes when the dynamics start with a random selected node for several values of coefficient of spontaneous rumor refutation $\gamma$.

\section{Conclusions}

Considering the rumor refutation mechanism, we divided it into two aspects: global and local, and analyzed them, respectively. In addition, we also considered the influence of individual activity rates on rumor propagation. According to their activity rate, they are divided into active and inactive groups. Active individuals can be infected by all spreaders, but inactive individuals can only be infected by active spreaders. Therefore, we improved the ISR model and obtained a model considering the global and local effects of the rumor refutation mechanism and the individual activity rates. Through the numerical simulation of the model, it is not difficult to see that individuals are more affected by the rumor propagation atmosphere of the whole society, but less affected by the people around them. This is because NGOs rumor refutation mechanisms are perceived to be unreliable. It can also be seen from the numerical simulation results that the strength of the refutation mechanism has largely prevented the large-scale rumor propagation. Therefore, we make recommendations as followings. First, we should strengthen the government's 
guidance and promptly refute rumors in order to control the further spread of rumors. Moreover, individuals should pay more attention to the channels or platforms of official information release. Finally, when implementing the rumor refutation mechanism, global and local rumor refutation should be combined to achieve the best rumor refutation effect. We noticed that the strength of the refutation mechanism spontaneously between people might be related to the personality of the individual. Our paper does not study this, but it can be used as a further research direction for our future work. Our work also shows that in the field of rumors propagation dynamics, control of rumor dynamics can be achieved by considering increasing efforts to refute rumors.

Author Contributions: Conceptualization, L.H.; methodology, L.H.; software, Y.Z.; validation, Y.Z.; formal analysis, L.H.; investigation, L.H. and Y.Z.; data curation, Y.Z.; writing-original draft preparation, Y.Z.; writing-review and editing L.H. and Y.Z.; visualization, Y.Z.; supervision, L.H. All authors have read and agreed to the published version of the manuscript.

Funding: This work was partially supported by the Program for Professor of Special Appointment (Eastern Scholar) at Shanghai Institutions of Higher Learning, and the Project for the Natural Science Foundation of Shanghai (21ZR1444100), and the Project for the National Natural Science Foundation of China (72174121, 71774111, 71871144, 71804047).

Institutional Review Board Statement: Not applicable.

Informed Consent Statement: Not applicable.

Data Availability Statement: Not applicable.

Acknowledgments: The authors are very grateful to the anonymous referees for their valuable comments and suggestions, helping them to improve the quality of this paper.

Conflicts of Interest: The authors declare no conflict of interest.

\section{References}

1. Ramos, M.; Shao, J.; Reis, S.D.; Anteneodo, C.; Andrade, J.S.; Havlin, S.; Makse, H.A. How does public opinion become extreme? Sci. Rep. 2015, 5, 10032. [CrossRef] [PubMed]

2. Kosfeld, M. Rumours and markets. J. Math. Econ. 2005, 41, 646-664. [CrossRef]

3. Huang, H.; Chen, Y.; Ma, Y. Modeling the competitive diffusions of rumor and knowledge and the impacts on epidemic spreading. Appl. Math. Comput. 2021, 388, 125536. [CrossRef] [PubMed]

4. Yin, F.; Lv, J.; Zhang, X.; Xia, X.; Wu, J. COVID-19 information propagation dynamics in the Chinese Sina-microblog. Math. Biosci. Eng. 2020, 17, 2676-2692. [CrossRef]

5. Daley, D.J.; Kendall, D.G. Epidemics and rumours. Nature 1964, 204, 1118. [CrossRef]

6. Maki-Thompson, D.P. Mathematical Models and Applications, with Emphasis on Social, Life, and Management Science; Prentice-Hall: Englewood Cliffs, NJ, USA, 1973; p. 492.

7. Newman, M.E.J.; Forrest, S.; Balthrop, J. Email networks and the spread of computer viruses. Phys. Rev. E Statal Nonlinear Soft Matter Phys. 2002, 66, 035101. [CrossRef]

8. Watts, D.J.; Strogatz, S.H. Collective dynamics of 'small-word' network. Nature 1998, 393, 440-442. [CrossRef]

9. Zanette, D.H. Dynamics of rumor propagation on small word networks. Phys. Rev. E 2001, 65, 110-126.

10. Moreno, Y.; Nekovee, M.; Pacheco, A.F. Dynamics of rumor spreading in complex networks. Phys. Rev. E 2004, 69, 066130. [CrossRef]

11. Nekovee, M.; Moreno, Y.; Bianconi, G.; Marsili, M. Theory of rumour spreading in complex social networks. Phys. A Stat. Mech Its Appl. 2007, 374, 457-470. [CrossRef]

12. Zhu, L.; Guan, G.; Li, Y. Nonlinear dynamical analysis and control strategies of a network-based SIS epidemic model with time delay. Appl. Math. Model. 2019, 70, 512-531. [CrossRef]

13. Xia, C.; Wang, L.; Sun, S.; Wang, J. An SIR model with infection delay and propagation vector in complex networks. Nonlinear Dyn. 2012, 69, 927-934. [CrossRef]

14. Liu, X.; Li, T.; Tian, M. Rumor spreading of a SEIR model in complex social networks with hesitating mechanism. Adv. Differ. Equ. 2018, 2018, 391. [CrossRef]

15. Zhao, L.; Wang, J.; Chen, Y.; Wang, Q.; Cheng, J.; Cui, H. SIHR rumor spreading model in social networks. Phys. A Stat. Mech. Its Appl. 2012, 391, 2444-2453. [CrossRef]

16. Yang, A.; Huang, X.; Cai, X.; Zhu, X.; Lu, L. ILSR rumor spreading model with degree in complex network. Phys. A Stat. Mech. Its Appl. 2019, 531, 121807. [CrossRef] 
17. Huo, L.; Cheng, Y. Dynamical analysis of a IWSR rumor spreading model with considering the self-growth mechanism and indiscernible degree. Phys. A Stat. Mech. Its Appl. 2019, 536, 120940. [CrossRef]

18. Zan, Y.; Wu, J.; Li, P.; Yu, Q. SICR rumor spreading model in complex networks: Counterattack and self-resistance. Phys. A Stat. Mech. Its Appl. 2014, 405, 159-170. [CrossRef]

19. Liu, C.; Zhou, L.X.; Fan, C.J.; Huo, L.A.; Tian, Z.W. Activity of nodes reshapes the critical threshold of spreading dynamics in complex networks. Phys. A Stat. Mech. Its Appl. 2015, 432, 269-278. [CrossRef]

20. Huo, L.; Ding, F.; Liu, C.; Cheng, Y. Dynamical analysis of rumor spreading model considering node activity in complex networks Complexity 2018, 2018, 1049805. [CrossRef]

21. Zhao, L.; Wang, X.; Wang, J.; Qiu, X.; Xie, W. Rumor-propagation model with consideration of refutation mechanism in homogeneous social networks. Discret. Dyn. Nat. Soc. 2014, 2014, 1-11. [CrossRef]

22. Zhang, N.; Huang, H.; Su, B.; Zhao, J.; Zhang, B. Dynamic 8-state ICSAR rumor propagation model considering official rumor refutation. Phys. A Stat. Mech. Its Appl. 2014, 415, 333-346. [CrossRef]

23. Zhang, Y.; Su, Y.; Li, W.; Liu, H.; Weigang, L. Modeling rumor propagation and refutation with time effect in online social networks. Int. J. Mod. Phys. C 2018, 29, 1850068. [CrossRef]

24. Einwiller, S.A.; Kamins, M.A. Rumor has it: The moderating effect of identification on rumor impact and the effectiveness of rumor refutation1. J. Appl. Soc. Psychol. 2010, 38, 2248-2272. [CrossRef]

25. Jia, F.; Cao, C. Dynamic analysis of a stochastic rumor propagation model with regime switching. Mathematics $2021,9,3277$. [CrossRef]

26. Zhao, L.; Qiu, X.; Wang, X.; Wang, J. Rumor spreading model considering forgetting and remembering mechanisms in inhomogeneous networks. Phys. A Stat. Mech. Its Appl. 2013, 392, 987-994. [CrossRef]

27. Dong, S.; Deng, Y.; Huang, Y. SEIR Model of rumor spreading in online social network with varying total population size. Commun. Theor. Phys. 2017, 68, 145-152. [CrossRef]

28. Zhu, L.; Zhao, H. Dynamical behaviors and control measures of rumour-spreading model with consideration of network topology. Int. J. Syst. Sci. 2017, 48, 2064-2078. [CrossRef]

29. Li, D.; Ma, J. How the government's punishment and individual's sensitivity affect the rumor spreading in online social networks. Phys. A Stat. Mech. Its Appl. 2017, 469, 284-292. [CrossRef]

30. Li, W.; Guo, T.; Wang, Y.; Chen, B. DR-SCIR public opinion propagation model with direct immunity and social reinforcement effect. Symmetry 2020, 12, 584. [CrossRef]

31. Wang, Y.; Wang, J. SIR rumor spreading model considering the effect of difference in nodes' identification capabilities. Int. J. Mod. Phys. C 2017, 28, 1750060. [CrossRef]

32. Xu, Y.; Mei, R.; Yang, Y.; Kong, Z. Modeling and analysis of rumor spreading with social reinforcement mechanism. Adv. Math. Phys. 2019, 2019, 7241021. [CrossRef]

33. Cheng, Y.; Huo, L.; Ma, L.; Guo, H. Dynamical behaviors and spatial diffusion in a psychologically realistic rumor spreading model. Int. J. Mod. Phys. C 2020, 31, 205003. [CrossRef]

34. Hu, Y.; Pan, Q.; Hou, W.; He, M. Rumor spreading model with the different attitudes towards rumors. Phys. A Stat. Mech. Its Appl. 2018, 502, 331-344. [CrossRef]

35. Chen, X.; Wang, N. Rumor spreading model considering rumor credibility, correlation and crowd classification based on personality. Sci. Rep. 2020, 10, 5887. [CrossRef] 